\title{
Sepsis caused by Myroides spp. associated with bronchopneumonia and co- infection with Pseudomonas aeruginosa and Proteus vulgaris: A case report and review of Myroides spp. infections.
}

\author{
Victoria Birlutiu1, Rares Mircea Birlutiu ${ }^{2 *}$ \\ ${ }^{1}$ Faculty of Medicine Sibiu, Lucian Blaga University of Sibiu, Academic Emergency Hospital Sibiu, Romania- \\ Infectious Diseases Clinic, Sibiu, Romania \\ ${ }^{2}$ Faculty of Medicine Sibiu, Lucian Blaga University of Sibiu, "FOISOR" Clinical Hospital of Orthopedics, \\ Traumatology and Osteoarticular TB Bucharest, Romania
}

\begin{abstract}
Background: Myroides spp. is considered an opportunistic germ, possibly pathogenic in immunocompromised patients, in whom it may cause varied clinical pictures in terms of severity, from skin and soft tissue infections, fasciitis, to urinary or respiratory infections, central nervous system infections, endocarditis etc. Few cases of sepsis caused by Myroides spp. have been described.

Case presentation: We present the case of an immunocompromised patient with diabetes mellitus, chronic renal failure, diagnosed with sepsis caused by Myroides spp. with a chronic leg ulcer as starting point, superinfected with Pseudomonas aeruginosa and Proteus vulgaris, and associating bronchopneumonia.

Conclusion: The presence of Myroides spp. or of other opportunistic pathogens in an immunocompromised patient, especially in those with renal failure, diabetes and persistent skin lesions, should be considered in cases with lack of response to commonly recommended antibiotics in these situations, as a possible emergence of an opportunistic pathogen that creates difficulties for treatment.
\end{abstract}

Keywords: Myroides spp., Sepsis, Bronchopneumonia, Co-infections, Biofilm.

Accepted on October 25, 2017

\section{Introduction}

Myroides spp. is an opportunistic infection-related pathogen in immunocompromised patients, most frequently being described in skin and soft tissue infections, rarely in pneumonia, bacteremia, venous-catheter related bacteremia, meningo-cerebral infections, endocarditis, and ventriculitis. The most common causes of associated immunosuppression are: diabetes mellitus, renal impairment, malignancy, and immunosuppressive therapy. Varied susceptibility to $\beta$-lactams, aminoglycosides, fluoroquinolones make it difficult to choose the first-line therapy. The association of an infection caused by Myroides with other bacteria in biofilm formation skin infections have not been described so far.

\section{Case Report}

We present the case of a 68 y old Caucasian male patient that has been hospitalized in the Cardiology Department for severe dyspnea and orthopnoea, chills, fever at home, coughing, and impaired general condition. Patient's medical history revealed anteroseptal myocardial infarction, atrial fibrillation, dilated cardiomyopathy, pulmonary hypertension, NYHA heart failure class IV, hypertension, insulin-dependent diabetes mellitus (diagnosed 20 y ago), diabetic nephropathy, chronic venous insufficiency CEAP C6. In the last two years, the patient had multiple admissions within the Dermatology Department for chronic bilateral leg ulceration, infected with a multidrugresistant Pseudomonas aeruginosa, the last admission being 3 months prior to this episode.

On physical examination upon admission, the following could be noticed: fever $38.9^{\circ} \mathrm{C}$, right shank ulcer of about $1 / 0.5 \mathrm{~cm}$, left leg skin graft (performed 6 months ago), bilateral leg stasis dermatitis, body mass index $38.16 \mathrm{~kg} / \mathrm{m}^{2}$, emphysematous chest, diffusely disseminated crackles, $\mathrm{SaO}_{2} 86 \%$, Heart Rate (HR) of 130 beats per min (bpm), permanent atrial fibrillation, systolic murmur grade III/VI in all auscultation sites, Blood Pressure (BP) of 150/80 $\mathrm{mmHg}$, hepatomegaly, and oliguria.

Laboratory investigations revealed the following alterations: Creactive protein $303.06 \mathrm{mg} / \mathrm{L}$ (references values 0-5), fibrinogen $793.9 \mathrm{mg} / \mathrm{dL}$ (references values 170-420), erythrocyte sedimentation rate $48 \mathrm{~mm} / \mathrm{h}$ (references values $0-15$ ), white blood cells $15.14 \times 10^{3} / \mu 1$, neutrophils $12.23 \times$ $10^{3} / \mu 1$, platelets $438 \times 10^{3} / \mu 1$, blood urea nitrogen $100 \mathrm{mg} / \mathrm{dL}$ (references values 18-55), creatinine $1.80 \mathrm{mg} / \mathrm{dL}$ (references values 0,7-1-3), and blood glucose $124 \mathrm{mg} / \mathrm{dl}$. Blood cultures 
taken on admission, from a central venous catheter, were positive for Myroides spp. (cultures for aerobic bacteria, incubated in BacT/ALERT ${ }^{\circledR}$ 3D Microbial Detection Systems (bioMerieux, France). Direct Sensitivity Test (DST) in automatic VITEK $^{\circledR} 2$ Compact 15 system (bioMerieux, France) revealed susceptibility to cefepime, ciprofloxacin, imipenem, pefloxacin, meropenem, minocycline, and resistance to aztreonam, amikacin, tobramycin, gentamicin, piperacillin, piperacillin/tazobactam, ticarcillin, ceftazidime, colistin, and trimethoprim/sulfamethoxazole. The MICs (minimum inhibitory concentrations) were assessed according to the European Committee on Antimicrobial Susceptibility Testing breakpoints. Bacteriological examination of shank ulceration was positive for Proteus vulgaris, sensitive to amikacin, cefepime, ciprofloxacin, gentamicin, pefloxacin, tobramycin, trimethoprim/sulfamethoxazole, meropenem and Pseudomonas aeruginosa, sensitive to amikacin, colistin, gentamicin, imipenem, meropenem, tobramycin, resistant to cefepime, ceftazidime, ciprofloxacin, pefloxacin, piperacillin, ticarcillin, ticarcillin/clavulanic acid, and trimethoprim/ sulfamethoxazole.

Chest radiography was performed and revealed the presence of numerous inhomogeneous opacities in both lungs, and minimal basal bilateral pleural effusions. Also, a chest computed tomography scan was performed, that concluded with the diagnosis of bronchopneumonia. The transthoracic echocardiogram revealed moderate mitral regurgitation and moderate tricuspid insufficiency, with circumferential pericardial fluid with a diameter of $12 \mathrm{~mm}$ (Figure 1).

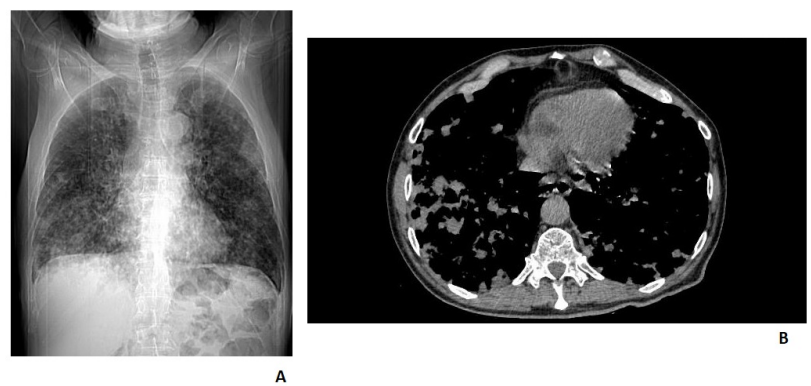

Figure 1. CT scan images (A: CT scout view. B: CT axial view).

Clinical data in conjunction with laboratory examinations supported the final diagnosis of sepsis caused by Myroides spp., bronchopneumonia, dilated cardiomyopathy, permanent atrial fibrillation, moderate pericarditis, moderate tricuspid insufficiency, moderate mitral regurgitation, NYHA heart failure class IV, stage 3 chronic kidney disease, insulindependent type 2 diabetes mellitus, leg ulcers superinfected with Pseudomonas aeruginosa and Proteus vulgaris.

The evolution was favourable under treatment with meropenem $2 \mathrm{~g} / \mathrm{d}$ for $12 \mathrm{~d}$, started on the $4^{\text {th }} \mathrm{d}$ of hospitalization, and replaced $4 \mathrm{~d}$ after with ceftazidime $3 \mathrm{~g} / \mathrm{d}$, after obtaining the results of sensitivity testing, associated to the treatment for the underlying diseases: digoxin, furosemide, spironolactone, acenocoumarol, and rimenidine. On discharge, the recommendation was to continue treatment with ciprofloxacin $2 \times 250 \mathrm{mg} / \mathrm{d}$, for another $7 \mathrm{~d}$.

\section{Discussions}

Myroides genus was known as Flavobacterium odoratum and belongs to the family Flavobacteriaceae [1], tribe Chromobacteriaceae. It is represented by non-fermentative gram-negative bacilli, non-motile, catalase-positive, oxidasepositive, yellow-pigmented because of the presence of flexirubin pigment [2] and fruity odor. Phenotypically, there are two different species, $M$. odoratus (which does not grow on MacConkey agar culture medium) and $M$. odoratimimus. In recent years, other 3 species were isolated from seawater [3-5], respectively $M$. marinus, $M$. profundi and $M$. pelagicus. It is considered that infection in humans is due to the takeover of bacteria from the environment, contaminated soil or water; in humans, Myroides spp. was isolated from blood, stool, urine, infected wounds; sometimes its role in pathogenesis is not very clear.

In immunocompromised patients, Myroides may be responsible for skin infections in patients with diabetes [6,7], urinary tract infections, lung infections [8-10], endocarditis [11], ventriculitis [12,13], bacteremia [14] or infections of the central nervous system. Rarely, there have been reported cases of infection in immunocompetent hosts $[12,13]$; the described cases were associated with hepatic, renal insufficiency, malignancies or co-infections with Oerskovia turbata in alithiasic cholecystitis [15].

So far, there has not been described any case of co-infections with other gram-negative bacteria, such as the case that we presented, which we believe that Myroides co-aggregated at the level of leg ulcer together with Proteus vulgaris and Pseudomonas aeruginosa to produce biofilm, allowing the multiplication and hematogenous dissemination of Myroides spp. The absence of the germ, from the bacteriological examinations of leg ulcers, does not exclude this entrance gate for bacteremia, the samples that were harvest on admissions were grown on usual growth medium. A growing on MacConkey agar medium was not used. Identification in a blood culture of an opportunist germ, commonly absent on the skin or mucous membranes, is sufficient to discuss about bacteremia with this germ. The patient had multiple admissions, which explains antibiotic resistance of Pseudomonas aeruginosa and Proteus vulgaris isolates. However, isolated Myroides strain was sensitive to carbapenems, fluoroquinolones, minocycline and cefepime, compared to other cases cited in the literature, that generally recorded resistance to $\beta$-lactams through chromosomally encoded the subclass B1 of metallo- $\beta$-lactamases [16] or variable susceptibility to aminoglycosides or quinolones [8,12,17-20]. Common susceptibility of isolated strains to meropenem allowed monotherapy in doses appropriate to renal impairment, recommending at discharge another $7 \mathrm{~d}$ of ciprofloxacin. Myroides susceptibility to antibiotics suggests a contamination subsequent to the last hospitalization, from the environment. 
Sepsis caused by Myroides spp. associated with bronchopneumonia and co-infection with Pseudomonas aeruginosa and Proteus vulgaris: A case report and review of Myroides spp. infections

\section{Conclusion}

The presence of Myroides spp. in immunocompromised patients, especially in those with renal failure, diabetes and persistent skin lesions, should be considered, and also other opportunistic pathogens, in cases with lack of response to commonly recommended antibiotics in these situations, as a possible emergence of an opportunistic germ that creates difficulties for treatment.

\section{Ethics Approval and Consent to Participate}

Written informed consent was obtained from the patient for publication of this case report and any accompanying images. The study was accepted by the Ethics Committee of the hospital and they encouraged publishing the article. A copy of the written consent is available for review by the Editor-inChief of this journal.

\section{Funding}

The authors received no funding for the manuscript preparation or for publishing the article.

\section{References}

1. Jooste PJ, Hugo CJ. The taxonomy, ecology and cultivation of bacterial genera belonging to the family Flavobacteriaceae. Int J Food Microbiol 1999; 53: 81-94.

2. Bernardet JF, Segers P, Vancanneyt M, Berthe F, Kersters K, Vandamme P. Cutting a Gordian knot: emended classification and description of the genus Flavobacterium, emended description of the family Flavobacteriaceae, and proposal of Flavobacterium hydatis nom. nov. (basonym, Cytophaga aquatilis Strohl and Tait 1978). Int J Syst Bacteriol 1996; 46: 128-148.

3. Yoon J, Maneerat S, Kawai F, Yokota A. Myroides pelagicus sp. nov., isolated from seawater in Thailand. Int J Syst Evol Microbiol 2006; 56: 1917-1920.

4. Zhang XY, Zhang YJ, Chen XL, Qin QL, Zhao DL. Myroides profundi sp. nov., isolated from deep-sea sediment of the southern Okinawa Trough. FEMS Microbiol Lett 2008; 287: 108-112.

5. Cho SH, Chae SH, Im WT, Kim SB. Myroides marinus sp. nov., a member of the family Flavobacteriaceae, isolated from seawater. Int J Syst Evol Microbiol 2011; 61: 938-941.

6. Endicott-Yazdani TR, Dhiman N, Benavides R, Spak CW. Myroides odoratimimus bacteremia in a diabetic patient. Proc (Bayl Univ Med Cent) 2015; 28: 342-343.

7. Motwani B, Krezolek D, Symeonides S, Khayr W. Myroides odoratum Cellulitis and Bacteremia: A Case Report. Infect Dis Clin Pract 2004; 12: 343-344.

8. Ktari SS, Mnif B, Koubaa M, Mahjoubi F, Ben Jemaa M, Mhiri MN, Hammami A. Nosocomial outbreak of Myroides odoratimimus urinary tract infection in a Tunisian hospital. J Hosp Infect 2012; 80: 77-81.
9. Yagci A, Cerikçioglu N, Kaufmann ME, Malnick H, Söyletir G, Babacan F. Molecular typing of Myroides odoratimimus (flavobacterium odoratum) urinary tract infections in a Turkish hospital. Eur J Clin Microbiol Infect Dis 2000; 19: 731-732.

10. Mei-Yu S, Wen-Liang Y, Che-Kim T. Urinary tract infection caused by myroides species: A case report. Journal of Microbiol Immunol Infect 2015; 8: 133.

11. Sharma S, Gupta A, Rao D. Myroides species: a rare cause of endocarditis. IJSS Case Rep Rev 2016; 2: 15-16.

12. Maraki S, Sarchianaki E, Barbagadakis S. Myroides odoratimimus soft tissue infection in an immunocompetent child following a pig bite: case report and literature review. Braz J Infect Dis 2012; 16: 390-392.

13. Benedetti P, Rassu M, Pavan G, Sefton A, Pellizzer G. Septic shock, pneumonia, and soft tissue infection due to Myroides odoratimimus: report of a case and review of Myroides infections. Infection 2011; 39: 161-165.

14. Kavita P, Ankur G, Abubakr B. Severe pneumonia due to an atypical organism in an immune-competent patient. Case Rep Int Med 2015; 2: 76-81.

15. Thomas M, Padmini SB, Govindan VK, Appalaraju B. Oerskovia turbata check for this species in other resources and Myroides species: Rare isolates from a case of acalculus cholecystitis. Ind J Med Microbiol 2007; 25: 297-298.

16. Mammeri H, Bellais S, Nordmann P. Chromosome-encoded beta-lactamases TUS-1 and MUS-1 from Myroides odoratus and Myroides odoratimimus (formerly Flavobacterium odoratum), new members of the lineage of molecular subclass B1 metalloenzymes. Antimicrob Agents Chemother. 2002; 46(11): 3561-7.

17. Crum-Cianflone NF, Matson RW, Ballon-Landa G. Fatal case of necrotizing fasciitis due to Myroides odoratus. Infection 2014; 42: 931-935.

18. Deepa R, Venkatesh K, Parveen JD, Banu ST, Jayalakshmi G. Myroides odoratus and Chryseobacterium indologenes: Two rare isolates in the immunocompromised. Indian J Med Microbiol 2014; 32: 327-330.

19. Green BT, Green K, Nolan PE. Myroides odoratus cellulitis and bacteremia: case report and review. Scand J Infect Dis 2001; 33: 932-934.

20. Elantamilan D, Valarie WL, Basabdatta C, Annie BK, Jyotismita R. Septicaemia caused by Myroides spp.-A case report. JMM Case Reports 2015; 2: 1-4.

\section{*Correspondence to}

Rares Mircea Birlutiu

Faculty of Medicine Sibiu

Lucian Blaga University of Sibiu

"FOISOR" Clinical Hospital of Orthopedics

Traumatology and Osteoarticular TB Bucharest

Romania 